\title{
QUANTITATIVE ANALYSIS BY UV-VIS DETECTION IN FLOW-ASSISTED SEPARATION TECHNIQUES FOR DISPERSED SAMPLES ${ }^{*}$
}

PART I: THEORY

\author{
PART II: EXPERIMENTAL TESTS WITHAPPLICATIONS \\ TO FIELD-FLOW. FRACTIONATION
}

\author{
Pierluigi Reschiglian", Andrea Zattoni, Giancarlo Torsi and \\ Dora Melucci \\ Department of Chemistry "G. Ciamician", University of Bologna \\ Via Selmi, 2 I-40126 Bologna (Italy) \\ *fax: +39 (0) 51209 9456; e-mail: reskv@ciam.umibo.it
}

\begin{abstract}
SUMMARY
Real samples are often in dispersed form and analytes in solution are frequently associated with other analytes in dispersion. Among analytical methods for nano- or micro-meter size dispersed samples, Field-Flow Fractionation has been the most applied flow-assisted separation technique. Hydrodynamic Chromatography, Size Exclusion Chromatography and even Capillary Electrophoresis have also recently spanned such an application range. For all these techniques, the UV-Vis detector is the workhorse. Dispersed analytes give rise to extinction of light even when absorption is absent. As a consequence, such detectors work more as turbidimeters than normal spectrophotometers. In order to convert the analytical signal for quantitative analysis, the validity of a Beer-Lambert like law in flow-through systems must be established for a turbidimetric response. In this paper we review the work done to extend the applicability of the Beer-Lambert law to measurements obtained by UV-Vis spectrometry for flow-assisted separation of dispersed analytes. In PART I we present the models to derive and apply the modified Beer-Lambert law, while in PARI II we discuss the results
\end{abstract}


obtained by comparing them with the theoretical values derived from the models. Some applications to quantitative particle size distribution analysis in field-flow fractionation are given.

Keywords: Dispersed Samples, Turbidity, Flow-assisted Separation Techniques, Field-Flow Fractionation

\section{CONTENTS}

INTRODUCTION 241

- Quantitative analysis in separation techniques for dispersed systems ... 241

- Flow-through UV-Vis spectroscopic analysis of dispersed systems .... 242

- Quantitative particle size distributions in field-flow fractionation ........ 243

PART I: THEORY ..................................................................... 244

- The Beer-Lambert-like law for heterogeneous flow-through systems .. 244

- Evaluation of the extinction coefficient of dispersed systems .............. 247

1. Calibration approach .............................................................. 247

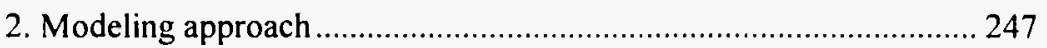

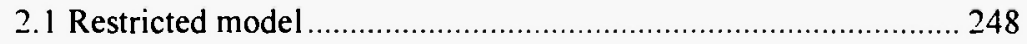

2.1.1 Predictive ability.......................................................... 250

2.1.2 Limitations and domain restriction ...................................... 250

- Quantitative particle size distribution in field-flow fractionation......... 253

PART II: EXPERIMENTAL TESTS WITH APPLICATIONS TO FIELD-FLOW FRACTIONATION.............................. 255

- Evaluation of the extinction coefficient by the calibration approach ... 255

- Evaluation of the extinction coefficient by the modeling approach ..... 259

- Particle size and sample mass distribution in field-flow fractionation .. 261

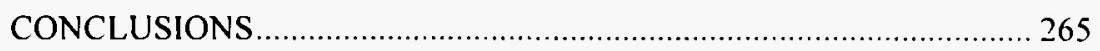

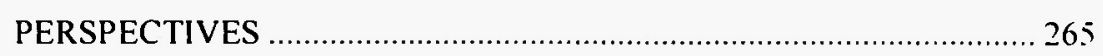

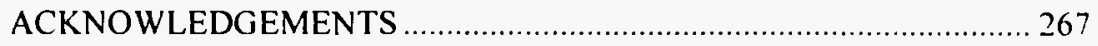

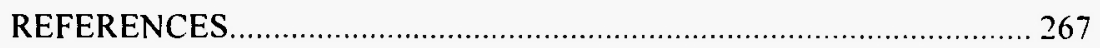




\section{INTRODUCTION}

\section{Quantitative analysis in separation techniques for dispersed systems}

In the typical instrumental arrangement of chromatographic-like techniques (including capillary electrophoresis) for the separation of dispersed samples, the detection has barely evolved from the use of classical UV-Vis detectors. While some on-line detectors more suitable for macromolecular and particulate matter analysis (like multi-angle laser light scattering (MALLS) or evaporative mass (EM) detectors) are available, UV$V$ is detectors remain the workhorses. In our opinion the reasons for such a conservative choice may be ascribed to at least one of the two following reasons. First, UV-Vis detectors are instruments that are readily available in any separation science laboratory. The use of such detectors, therefore, does not require an extra-cost when a separation technique, generally used for the analysis of molecular species, is applied to dispersed samples. Secondly, standard UV-Vis detectors are low-cost when compared to specific laser scattering detectors. They also require less maintenance. An extensive approach to quantitative aspects in the analysis of dispersed systems by separation techniques has been up to now delayed just because the use of standard UV-Vis detectors does not easily give quantitative answers. In fact, the main disadvantage when this type of detector is used for quantitative analysis of dispersed systems lies in the complex dependence of the analytical response on experimental conditions (e.g., sample size and optical properties, mobile phase composition). With particulate matter eluted from the separation device (the column in size exclusion or hydrodynamic chromatography, the capillary in electrophoresis, the channel in field-flow fractionation) standard detectors for liquid chromatography act as turbidimeters. In order to extract quantitative information from a separative run, one needs, thus, to work out a useful, hopefully simple relationship between the detector signal and the eluted mass (or concentration). The absorbance given by the Beer-Lambert (B.-L.) law has always been the preferred choice for quantitative analysis in UV spectroscopy. If a B.-L.-like expression holds true also for UV-Vis turbidity measurements, quantitative analysis of dispersed analytes can be made once the spectroscopic constant is obtained. 


\section{Flow-through UV-Vis spectroscopic analysis of dispersed systems}

The general solution for turbidimetry in flow-through systems thus requires to assess the validity of a B.-L.-like law for dispersed systems, and then to determine the proportionality constant for the same law (spectroscopic constant). We have shown that the derivation of the B.-L. law for the determination of sample amount in homogeneous flow-through systems can also be applied to dispersed samples /1/. It is, moreover, worthwhile to point out that such a derived B.-L.-like law can be used for standardless analysis of eluted, dispersed samples $/ 2 /$. A standardless method of analysis is defined as a method through which the analytical signal can be related to the concentration or quantity of the analyte from a single measurement. Working in standardless mode can thus allow for the direct and immediate conversion of the analytical signal into concentration or mass of the dispersed analyte while it is eluted from a separation device. Standardless analysis, however, necessarily requires a method either to determine experimentally or to predict the spectroscopic (turbidimetric) constant (the extinction coefficient). Experimental determination of the extinction coefficient can be performed through calibration with known amounts of particulate standards, as already shown by us $/ 1,2 \%$. Prediction of the extinction coefficient of dispersed particles can generally be obtained by means of the scattering theory given by Mie /3/. However, extended applications of the Mie theory have become affordable only recently because the Mie approach requires a huge amount of numerical work and the theory itself is quite complicated. Nevertheless, since Mie theory is very general, different theoretical, approximate approaches to the evaluation of the extinction properties of dispersed particles can be found in the literature, which can be applied within limited ranges of particle size /3/. Following a simpler, semi-empirical model that can be considered an approximation of the Mie theory for micronsize particles we have found that, within some limitations and approximations, there are specific ranges of experimental variables for which the extinction coefficient of dispersed, micronsize particles can be determined. We have found that the information gain that can be reached by means of such a modeling approach is worthwhile even if limited to a restricted range of experimental variables $/ 4 /$. 


\section{Quantitative particle size distributions in field-flow fractionation}

Particle size distribution (PSD) has recently been considered as one of the most outstanding topics in Analytical Science. In our opinion this should not be considered as an overstatement because most of the analytes from real samples are themselves either in dispersed form or in dispersed matrices. For dispersed systems, the size distribution is a feature as fundamental as the molecular weight of a molecular analyte. For instance, the characterization of the size of sample particles is routine and an essential part of overall quality control procedures. In the medical field, for particles used as drug carriers the size is a critical performance factor (e.g. liposomes). In the food industry, the alcoholic yield from fermentation of starch and even the taste of chocolate depends on the size of the particles of which these samples are composed. Before discussing our quantitative method for determining particle size, it is necessary to review briefly the definition of size distribution. If all particles of a given sample were spherical in shape, the only size parameter would be the diameter. In most real cases of irregular particles the size is usually expressed in terms of the diameter of a sphere equivalent to the particle with regard to some property. Particles of a dispersed sample are never perfectly identical in size and shape. A spread around the mean (distribution) is found. Such a spread is often described in terms of standard deviation. However, a frequency function, or its integrated (cumulative) distribution function more properly defines not only the spread but also the shape of such a spread around the mean value. This is commonly referred to as the Particle Size Distribution (PSD) profile of the dispersed sample.

An examination of the technical literature and trade publications indicates that a wide variety of instruments are commercially available for PSD analysis. Most of these techniques are not separation methods. However, because particulate dispersions are often highly complex in terms of polydispersity index, multimodal size distribution and density, it is hardly possible to obtain an accurate determination of their size distribution without the use of separative methods. Size exclusion chromatography, hydrodynamic chromatography and field-flow fractionation (FFF) are separation, flow-assisted chromatography-like methods for PSD analysis. In this review the proposed model for quantitative analysis is applied in standardless mode to quantitative PSD /2/. 


\section{PART I: THEORY}

\section{The Beer-Lambert-like law for heterogeneous flow-through systems}

The Beer-Lambert law takes the well-known form

$$
A=\varepsilon b c
$$

It is intrinsically well suited for quantitative analysis in UV-Vis spectroscopy because only one term $\left(\varepsilon\right.$, absorptivity $\left.\left[\mathrm{I} \mathrm{cm}^{-1} \mathrm{~mol}^{-1}\right]\right)$ must be theoretically or experimentally determined to relate the analytical signal $(A$, absorbance $[-])$ to concentration $\left(c,\left[\mathrm{~mol} \mathrm{l}^{-1}\right]\right)$ via the pathlength of the optical cell $(b,[\mathrm{~cm}])$.

In UV detectors, in flow-assisted separation techniques the concentration is not constant along the cell pathlength. However, all the assumptions for the validity of Eq. 1 are retained except that of sample homogeneity. However, if we assume that homogeneity is maintained in planes perpendicular to the optical beam, with the detector cell of constant cross-section, Eq. 1 then takes the form $/ 5 /$

$$
A(t)=\frac{10^{3} \cdot N(t)}{S}
$$

where $A(t)$ is the absorbance at time $t[\mathrm{~s}], S\left[\mathrm{~cm}^{2}\right]$ is the cross section of the detector cell, and $N(t)$ [mol] the number of moles present at time $t$ inside the cell. The $10^{3}$ factor is used for retaining the numerical value of $\varepsilon$ available from literature in the case of UV-Vis measurements.

The number of analyte moles present in the cell can be conveniently calculated with a convolution integral /5/

$$
N(t)=\int_{0}^{t} S\left(t^{\prime}\right) R\left(t-t^{\prime}\right) d t^{\prime}
$$

where $S(t)$ is the source function, $R(t)$ the normalized removal function and $t^{\prime}$ a dummy variable.

From the properties of the convolution integral we can write 


$$
\int_{0}^{\infty} N(t)=\int_{0}^{\infty} S(t) d t \int_{0}^{\infty} R(t) d t
$$

which can be written, after the substitution of $N(t)$ from Eq. 2, in the form

$$
S \int_{0}^{\infty} A(t)=10^{3} \varepsilon \int_{0}^{\infty} S(t) d t \int_{0}^{\infty} R(t) d t
$$

and after integration

$$
S \overline{\mathrm{A}}=10^{3} \varepsilon N_{0} \tau
$$

where $\overline{\mathrm{A}}[\mathrm{s}]$ and $N_{\theta}[\mathrm{mol}]$ are, respectively, the peak area and the total number of moles of the analyte that is eluted from the separation device and carried through the detector cell, and $\tau[\mathrm{s}]$ is the average time spent by a molecule of the analyte in the optical beam.

If the flow rate of the mobile phase in a given flow-assisted separation technique is constant, then $\tau$ can be defined as

$$
\tau=\frac{S b}{F}
$$

where $F\left[\mathrm{~cm}^{3} \mathrm{~s}^{-1}\right]$ is the flow rate.

The final B.-L. expression for UV-Vis detectors then has the form

$$
\overline{\mathrm{A}} F=10^{3} \varepsilon b N_{0}
$$

Equation 8 is a little more complicated than Eq. 1 but very easily implemented because both $F$ and $\bar{A}$ can be easily and accurately measured in flow-assisted separation techniques. If the area of the peak is not given in time units, as required by Eq. 8 , the conversion factor can be measured in different ways $15,6 /$. The above derivation demonstrates that the B.-L. law for flow-through systems (Eq. 8) can be considered equivalent to the B.-L. law for static, homogeneous systems (Eq. 1). In both cases, quantitative analysis can be obtained from a single measurement of the analytical signal.

When the sample flowing through the spectrophotometer cell is a dispersion of particles, even when absorption is absent, extinction of light 
occurs. In this case the detector works rather as a turbidimeter and the analytical signal ("absorbance") $A$, still defined as $\log \left(P_{0} / P\right)$, with $P_{0}$ and $P$ [erg s $\mathrm{cm}^{-1}$ ] the radiant powers exiting the cell. As a consequence, in order still to use $A$ for quantitative analysis, we have investigated whether it is still proportional to the concentration of the particulate sample. In other words, the validity of the above derived B.-L. law for flow-through systems can be ascertained for the turbidimetric signal.

From the definition of turbidity $T$ [ $\left.\mathrm{cm}^{-1}\right]$

$$
T=\frac{1}{b} \ln \frac{P_{0}}{P}
$$

we get the simple relationship

$$
A=\frac{T b}{\operatorname{In} 10}
$$

which states that turbidity $T$ is related to absorbance $A$ just through constants. Hence, the validity of Eq. I means the following relationship holds true even when the UV-Vis detector operates as a turbidimeter

$$
A=K b c
$$

where $c\left[\mathrm{~g} \mathrm{~cm}^{-3}\right]$ is the concentration and $K\left[\mathrm{~cm}^{2} \mathrm{~g}^{-1}\right]$ is the extinction coefficient, that is the proportionality constant which must be independent of c. This hypothesis has been validated by us and it is here described in PART II $/ 1,2 /$. Once verified that Eq. 11 is true, it can be used in a similar way as the B.-L. law for molecular absorbers in solution. As a consequence, the extension of the B.-L. law to flow-through spectrophotometric cells represented by Eq. 8 can be applied to fractionated particulate samples as well. In fact, the same mathematical procedure as the one used to get Eq. 8 from Eq. 1 can be applied to derive the relationship for the turbidimetric signal with flow-through cells

$$
\overline{\mathrm{A}} F=\nVdash b m_{0}
$$


where $\overline{\mathrm{A}}$ is the area of the turbidimetric peak and $m_{0}[\mathrm{~g}]$ is the mass of the particulate matter that was carried through the detector cell. Equation 12 does not require further validation since it has been validated already for the corresponding Eq. 8. The only difference lies in the absorptivity term $(\varepsilon)$ in Eq. 8 that is replaced by the extinction coefficient $(\mathcal{X})$ in Eq. 12. Equation 12 can, therefore, be applied to the standardless method. It is necessary only that particles are of the same type and dispersed in the same dispersing medium as those used for the experimental evaluation of $\mathcal{X}$. The effect of the dispersing medium in the evaluation of $\mathcal{K}$ is described in PART II.

\section{Evaluation of the extinction coefficient of dispersed systems}

\section{Calibration approach}

A calibration plot ( $A$ vs. $C$ ) can confirm the independence of the extinction coefficient $(\mathcal{K})$ from the analyte concentration $(c)$. At the same time, it can give the value of $\mathcal{K}$ from the slope, provided that $b$ is accurately known from independent measurements. The value of $b$ may be obtained by applying Eq. 1 to a spectroscopic standard of well-known absorptivity $\varepsilon$. The main advantage of the calibration approach to obtain $\mathcal{K}$ is that no specific knowledge of detector optics is needed. As mentioned above, the main disadvantage of this approach lies in the fact that, for the calibrations, one must use particles identical to those present in the sample. For real samples this is, in fact, a situation rarely encountered.

\section{Modeling approach}

The above procedure allows for quantitative analysis by means of a single, flow-through measurement with the value of the extinction coefficient $\mathcal{K}$ determined by calibration. As mentioned in the INTRODUCTION, the extinction coefficient can also be calculated from models. We did this by modeling the optical behavior of a particle in a beam of light $/ 1,4 /$.

In order to find a relationship through which the extinction coefficient $\mathcal{K}$ can be calculated from sample properties and instrumental parameters, we first restrict the case to spherical, monodispersed particles, for which we have the expression for turbidity /1/

$$
T=\pi a^{2} N Q
$$


where $a[\mathrm{~cm}]$ is the particle radius, $N\left[\mathrm{~cm}^{-3}\right]$ the number of particles per unit volume, and $Q[-]$ is called the extinction efficiency. This quantity is related to the way by which the light interacts with a particle and represents the ratio between the light extinction cross-section and the geometrical cross-section of the particle /3/. For the present case of spherical, monodispersed particles, $N$ is simply related to concentration $c$ as

$$
N=\frac{3 c}{4 \pi a^{3} \rho}
$$

where $\rho\left[\mathrm{g} \mathrm{cm}^{-3}\right]$ is the particle density. By combining Eqs. 10,13 and 14 we finally get the expression for the extinction coefficient as

$$
\mathcal{K}=\frac{3}{4 \operatorname{In} 10} \frac{Q}{\rho a}
$$

from which calculation of $\mathcal{K}$ is immediate provided we can find a reliable and accurate method for evaluating the extinction efficiency $Q$.

Equation 15 allows for the determination of the extinction coefficient of spherical particles of constant size (monodispersed particles). However, it holds true also for particles having a finite standard deviation in size around the mean (polydispersed particles) and for particles of irregular shape. It is known from the literature that polydispersed particles behave as monodispersed particles with apparent particle radius equal to one half of their volume-surface mean diameter (otherwise called the Sauter mean, $D_{32}$ ) 17\%. On the other hand, if the particles are not spherical they behave as though they have an average cross-section equal to one fourth of their surface area $13 \%$

\section{I Restricted model}

As already mentioned in the INIRODUCTION, the Mie theory can be a more general approach for the calculation of $Q / 3 /$. However, if we restrict our treatment to a particular range of particles, a restricted model can be applied. This model has already been proposed and tested by us $/ 4,8 /$. The assumptions are:

1) spherical, compact, non-absorbing particles of diameter larger than the incident wavelength $\lambda[\mathrm{cm}]$ 
2) refractive index of the particle real in value (i.e. particle without absorption in the UV-Vis region)

3) relative refractive index of the particle with respect to the dispersing medium $(\mu[-])$ close to unity

The above conditions can be summarized by the following expression

$$
\begin{gathered}
\mathrm{P}=\frac{4 \pi a}{\lambda}(\mu-1) \geq 2.5 \\
1<\mu<1.25
\end{gathered}
$$

where $\mathrm{P}[-]$ is the phase factor $/ 3 /$.

If particle features fulfill the above assumptions, we may consider the optical behavior of the particle as the combination of two independent contributions:

1) geometric light obstruction

2) low-angle light scattering (otherwise called Fraunhofer diffraction)

1) The contribution to $T$ given by geometric light obstruction is equal to $\pi a^{2} N$. That is, the light that a compact particle in the detector cell removes by geometrical obstruction cannot obviously reach the sensor at all. This is equivalent to a contribution to $Q$ equal to 1 (Eq. 13).

2) The scattered light is, under the above conditions given by Eqs. 16-17, always scattered at low angle (Fraunhofer diffraction), no matter what kind of particle is present. This implies a contribution to $T$ larger than in 1), whose limiting factor tends also equal to $\pi a^{2} N$. This is equivalent to a contribution $Q=1$ for particles much larger than the incident wavelength $(a>10 \lambda)$ (Eq. 13).

A result of this model is that a large spherical particle removes incident light with an overall extinction efficiency $Q=2$, that is twice what it can intercept. Because of the apparent paradoxical character of this optical behavior, the effect was called the extinction paradox $/ 3 /$. However, the extinction paradox holds true only if the detector is at infinite distance from the particle. When the distance between the particle and the detector sensor is very high compared to the detector size, only the light having a direction parallel to the incident light can reach the sensor, while all diffracted light is 
prevented from reaching it. Diffracted light goes towards the sensor with rays at low scattering angle $(\omega)$ with respect to the direction of the beam. We shall define the acceptance angle $(\theta)$ as the solid angle of the reception cone of the detector. It is known that most UV-Vis detectors have $\theta<5^{\circ} / 9 \%$. As a consequence, at least part of the diffracted light can be captured within the reception cone of the sensor. Its contribution to $Q$ is, thus, between 0 and 1 . If the light is diffracted at $\omega<\theta$, it is totally captured by the sensor and it contributes nothing to the extinction efficiency $Q$. The sum of the diffractive contribution and the obstructive contribution therefore results in an overall value of the extinction efficiency in the range $\mathrm{l}<Q<2$.

The procedure to predict the overall value of $Q$ as a function of $\mathrm{P}$ and $\theta$, within the limiting conditions reported above, has been derived by us from a semi-empirical model given in literature to describe the $Q$ function, which is valid for compact, spherical and non-absorbing particles $/ 10,11$.

\subsubsection{Predictive ability}

In Figure 1 (adapted from /4/) the predictive ability of the restricted model is tested by determining the dependence of the extinction efficiency for compact polystyrene particles. In Figure la) the ratio between the extinction efficiency $Q$ and the extinction efficiency at $\theta=0^{\circ}\left(Q_{0}\right)$ for a given value of $a / \lambda$ is shown as a function of $\theta$. From this curve it is evident that the extinction efficiency $Q$ rapidly decreases with increasing acceptance angle. In fact, at a $\theta$ value of only $3^{\circ}$, the $Q$ value is nearly half of the extinction efficiency at $\theta=0^{\circ}$. Moreover, in Figure $1 \mathrm{~b}$ ), it is shown that the rippling nature of $Q$ is dampened with increasing $a / \lambda$ and a constant, unit value of $Q$ is rapidly approached at higher acceptance angles. From these findings we can see that the acceptance angle is one of the most critical parameters to take into account for turbidity measurements with UV-Vis detectors. It is evident that the best choice is, for a given particle radius $(a)$, the use of a detector with the highest acceptance angle $(\theta \quad$ ), at the lowest possible wavelength $(\lambda)$. Experimental results for model predictability with respect to standard, polystyrene particles are reported in PART II.

\subsubsection{Limitations and domain restriction}

Through this restricted model we have plotted $Q$ vs. P and $\theta$ and we have demonstrated that $Q$ can be predicted in a broad range of instrumental and analysis conditions, as well as for a broad range of particle features (Fig. 2, adapted from /4/). At the same time we have shown that, for compact 

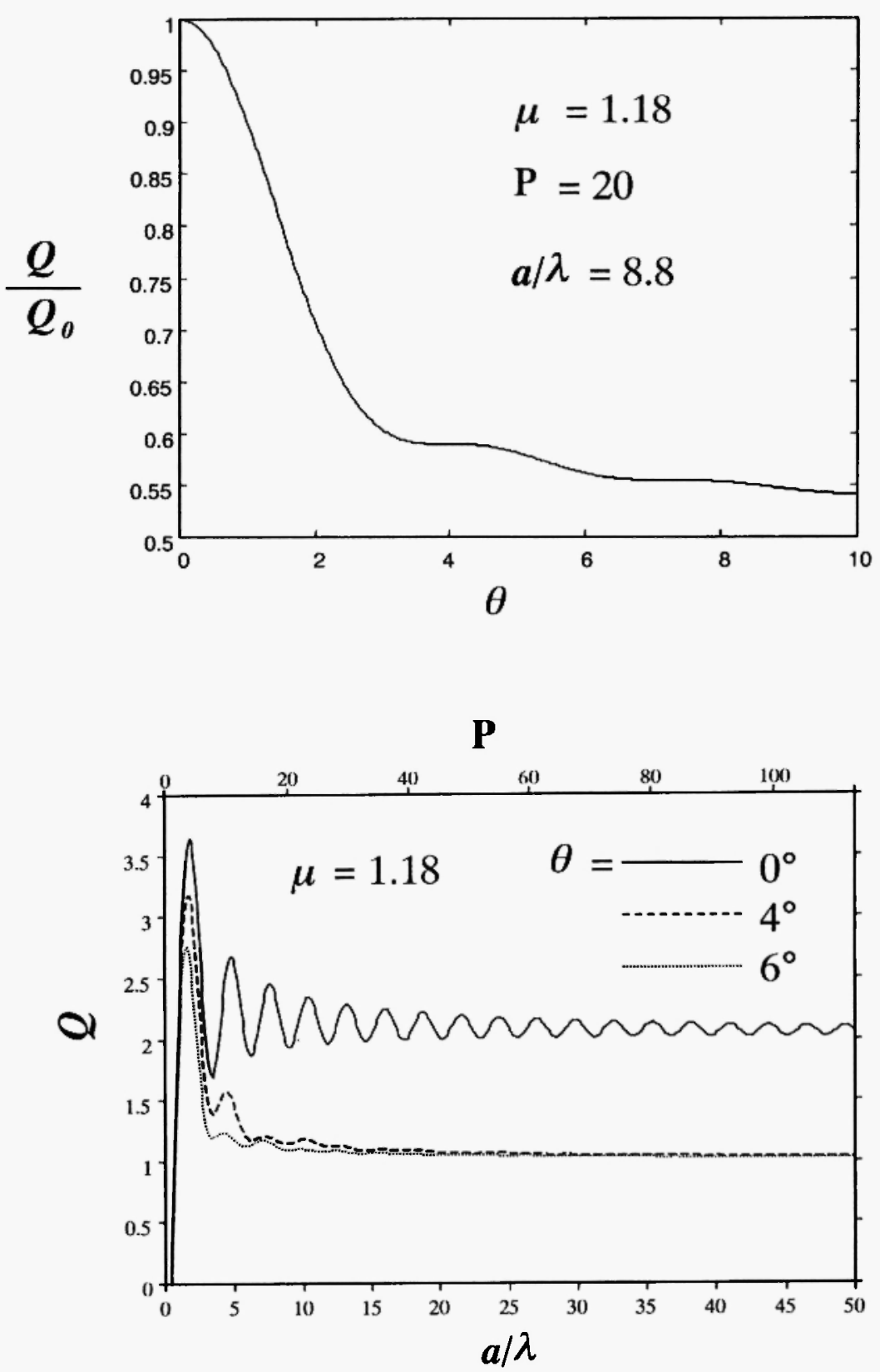

Fig. 1: Dependence of the effective extinction efficiency $(Q)$ on experimental variables: functions calculated for compact, spherical polystyrene particles. a) Ratio between $Q$ and $Q_{0}$ vs. the acceptance angle $\theta$; b) $Q$ vs. P and $a / \lambda$ on changing $\theta$ 


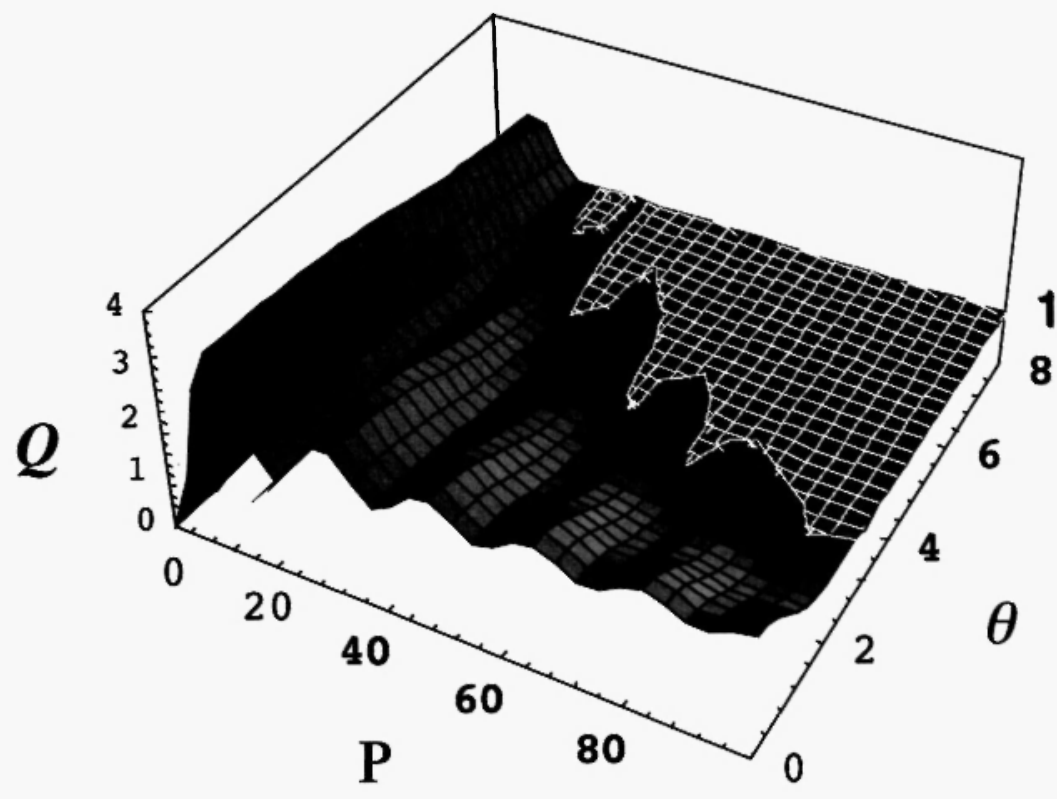

Fig. 2: $3 \mathrm{D}$ plot of the extinction efficiency $Q$ vs.P and $\theta$

particles, a wide area of the surface variables exists where $Q=1$. This area corresponds to the highest values of $\mathrm{P}$ and $\theta$. This means that in most practical cases we have the condition $\mathcal{E}=1$ fulfilled for a selected range of experimental and instrumental conditions. In other words, there is a given, and relatively broad range of conditions within which $Q=1$ can be predicted without the need for a strict knowledge of detector optics, particle features, and analysis conditions (see Eq. 16). This is of valuable practical relevance because it makes the calculation of the extinction coefficient $\mathcal{K}$ particularly straightforward. Its value is inversely proportional to the particle radius $(a)$, which must be, therefore, accurately known (Eq. 15). However, the evaluation of particle size cannot be thought of as a limiting factor of the modeling approach here described because it can be determined by an independent technique or directly obtained from measurements in the flowassisted separation of particles, as in the case of FFF.

One of the assumptions for the restricted model is that particles are compact. However, the model can also be adapted for non-compact (i.e. porous) particles. Porous particles are poorly refractive (relative refractive index $(\mu)$ very close to 1 ) and, thus, the refracted light captured within the 
acceptance cone of the spectrophotometer can be subtracted from the total extinction efficiency. We have implemented the restricted model with the correction term given in literature /12/ for the calculation of $Q$ for porous, micronsize silica particles $/ 8 \%$. Results on the adaptability of the modeling approach to porous particles are reported in PART II.

\section{Quantitative particle size distribution in field-flow fractionation}

The interesting aspect of FFF for PSD studies is that PSD profiles can be obtained by numerical transformation of the FFF elution profile. This is because in FFF the conversion from retention times to particle size can be obtained from first principles or from calibration with standards of known size /13/. However, without an approach to convert the UV detector signal into concentration or mass of the eluted sample, no quantitative, FFF-based PSD analysis was possible. In fact, PSD in FFF were reported as frequency functions proportional to mass rather than frequency distributions of real mass $/ 13 /$. With the approach here described we have shown that, when applied in standardless mode, we can directly and simultaneously obtain the particle size and the cumulative mass distributions from the digitized fractogram (the analytical signal vs. the retention volume). From the latter distribution one can obtain the total sample recovery. We called this quantitative PSD Particle Size and sample Amount Distribution (PSAD) /2/.

Particle size distribution curves as mass frequency functions that are just proportional to particle mass $\left(F_{n, i}\left[\mathrm{~cm}^{-1}\right]\right)$ can be easily obtained from digitized FFF signals (fractograms) $y_{i}\left(V_{r, i}\right)$ converted into a function of particle diameter $d_{i}[\mathrm{~cm}]$ as described by us in $/ 14 /$.

We can write

$$
F_{\mathrm{m} \dot{p}} \propto(\mathrm{UV} \text { signal a.u. }) d_{i} \cdot \frac{\delta V_{r \dot{p}}}{\partial d_{i}}
$$

where $\delta V_{r i}\left[\mathrm{~cm}^{3}\right]$ and $\delta d_{1}[\mathrm{~cm}]$ are the differences in retention volume and particle diameter between the $i$ th and the $(i-1)$ th digitized point of the fractogram. The expression for the PSAD can be obtained if we substitute into Eq. 18 the relationship to convert the detector signal to particle mass via the extinction coefficient $\mathcal{K}$ We shall examine now how to obtain this expression when the extinction coefficient is determined by either the modeling approach or the calibration approach. 
When the extinction coefficient is predicted by the modeling approach on the basis of a known value of $Q$ (Eq. 15), $F_{m . i}$ can be transformed into the real mass distribution $f_{m, ~}(d)$ [ $\left.\mathrm{g} \mathrm{cm}^{-1}\right]$ directly from the expression of turbidity in Eq. 10 when combined with Eq. 11. This yields

$$
\begin{gathered}
T_{i}=2.303 \mathcal{K}_{i} \frac{\delta m_{i}}{\delta V_{r, i}} \\
f_{\mathrm{m} \downarrow}(d)=\frac{\delta m_{i}}{\delta d_{i}}=\frac{T_{i}}{2.303 K_{i}} \cdot \frac{\delta V_{r i}}{\delta d_{i}}
\end{gathered}
$$

Integration of Eq. 20 gives the cumulative distribution of sample amount as a function of size with its asymptotic value giving the total sample recovery. The integrated expression plotted as cumulative percent distribution also gives the size distribution percentiles $\left(d_{10}, d_{50}, d_{90}\right)$.

When the extinction coefficient is experimentally determined by the calibration approach, $\mathcal{K}$ expresses an averaged value over particle size. In the cases for which $Q$ can be assumed to be constant (e.g. in the flat surface response in Fig. 2) the expression for $f_{\text {in }}(d)$ can be obtained as a function of the experimental extinction coefficient. The final expression can be derived by integrating $f_{\text {in.i }}(d)$ with respect to particle diameter $d$

$$
\int_{0}^{\infty} f_{\mathrm{m}, \mathrm{i}}\left(d_{i}\right) \delta d_{i}=m_{0}
$$

By multiplying and dividing Eq. 20 for the expressions of $m_{0}$ respectively in Eq. 12 and Eq. 21 we get

$$
\hat{J}_{m, i}(a)=\frac{\frac{T_{i}}{2.303 K_{i}} \frac{\delta V_{r \dot{i}}}{\delta d_{i}}}{\int_{0}^{\infty} f_{\mathrm{m}, \mathrm{i}} \delta d_{i}} \frac{\overline{\mathrm{A}} F}{K b}
$$

By substituting in the above equation the expression for $f_{\text {in, }}(d)$ in Eq. 20 with the $i$ th value of the extinction coefficient $\mathcal{K}_{i}$ expressed as in Eq. 15 we get 


$$
f_{m \dot{j}}(d)=\frac{T_{i} d_{i} \frac{\delta V_{r \dot{i}}}{\delta d_{i}}}{\int_{0}^{\infty} T_{i} d_{i} \frac{\delta V_{r \dot{p}}}{\delta d_{i}} \delta d_{i}} \frac{\overline{\mathrm{A}} F}{\kappa b}
$$

\section{PART II:}

\section{EXPERIMENTAL TESTS WITH APPLICATIONS TO FIELD-FLOW.FRACTIONATION.}

\section{Evaluation of the extinction coefficient by the calibration approach}

In PART I we have explained that in order to apply the B.-L.-like law to quantitative analysis of particles fractionated by flow-assisted techniques, it is first required to demonstrate that the analytical signal from the UV-Vis detector $(A)$ depends linearly on the analyte concentration (c). This is equivalent to demonstrating that the extinction coefficient $\mathcal{K}$ in Eq. 11 is constant with respect to $c$. We confirmed the linearity by directly filling the detector cell with dispersions of particles at different, known concentrations 11/. Each concentration corresponds to a constant value of $A$ and a regression analysis of the $(c, A)$ data demonstrates that a straight line with null intercept is the best fitting equation for the experimental points. The concentration range was chosen to include the transient, actual $A$ values obtained during a typical flow-assisted separation run, as in the FFF analysis of micronsized particles. Linearity can also demonstrate the absence of multiple scattering, which is known to be the main source of deviations from linearity in turbidity measurements at high concentration $/ 3 /$. Two examples are shown here in Figs. 3, 4 (adapted from /15/). Linearity is clearly demonstrated, with null intercept $/ 1 /$. It should be noted that Figs. 3, 4 correspond, respectively, to porous and compact particles. Hence the validity of Eq. 11 is confirmed no matter whether the particles are compact or not, as discussed in PART 1.

We focus now on the effects that the dispersing medium composition has on the extinction coefficient. Figure 5 (adapted from $/ 2 /$ ) shows plots for the determination of the extinction coefficient for the same type of spherical silica particles in various media. The samples are in aqueous media with, respectively, no surfactant $\left(1: \mathrm{NaN}_{3} 3.1 \mathrm{mM}\right)$, non-ionic surfactant at the same ionic strength (2: TRITON X-100 $0.1 \% ; \mathrm{NaN}_{3} 3.1 \mathrm{mM}$ ), and anionic 


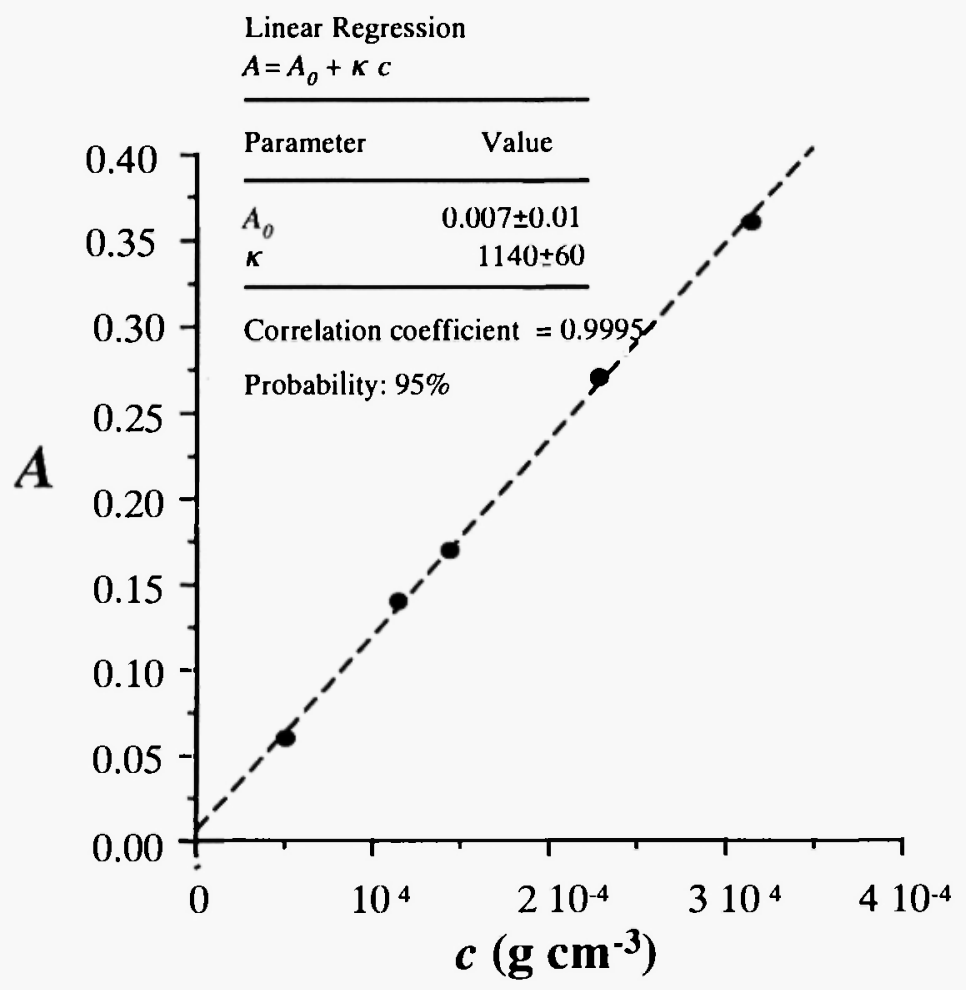

Fig. 3: $A$ vs. $c$ for dispersions of spherical silica particles in Triton $\mathrm{X}-100$ $0.1 \% \mathrm{v} / \mathrm{v}+\mathrm{NaN}_{3} 3.1 \mathrm{mM} . \lambda=330 \mathrm{~nm} . a=2.5 \mu \mathrm{m}$

surfactant alone at ionic strength two orders of magnitude lower (3: sodium dodecyl sulphate (SDS) $3.1 \quad 10^{-5} \mathrm{M}$ ). It is evident that for the cases of no surfactant $\left(\mathrm{NaN}_{3} 3.1 \quad 10^{-3} \mathrm{M}\right)$ and SDS added to the dispersing media the extinction coefficients do not significantly differ in value, even at different values of the ionic strength. However, for the dispersing medium containing non-ionic surfactant TRITON X-100 $0.1 \%$ and ionic strength equal to 3.1 $\mathrm{mM}$ (in $\mathrm{NaN}_{3}$ ) the value of $\mathcal{K}$ increases by a factor of two. We ascribed the above finding to either a different adsorption amount, a different dispersion state (micelles) or adsorption mechanisin of the surfactant adsorbed on particles. Whether and to what extent the surfactant TRITON X-100 can be adsorbed on particles has been established by us by means of indirect experiments $2 \%$. We have shown that a significant change of the optical properties of silica spheres occurs when TRITON X-100 is added beyond its 


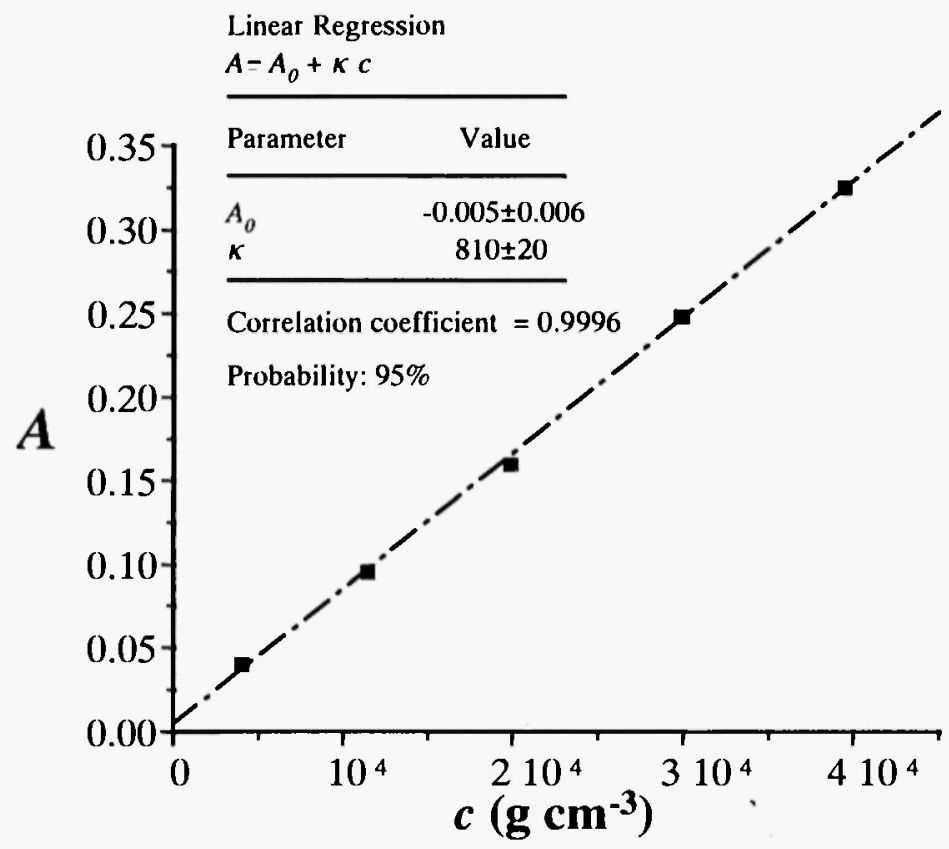

Fig. 4: $A$ vs. $c$ for dispersions of compact, spherical polystyrene particles in $\mathrm{NaCl} 3.1 \mathrm{mM} . \lambda=520 \mathrm{~nm} . a=3.05 \mu \mathrm{m}$

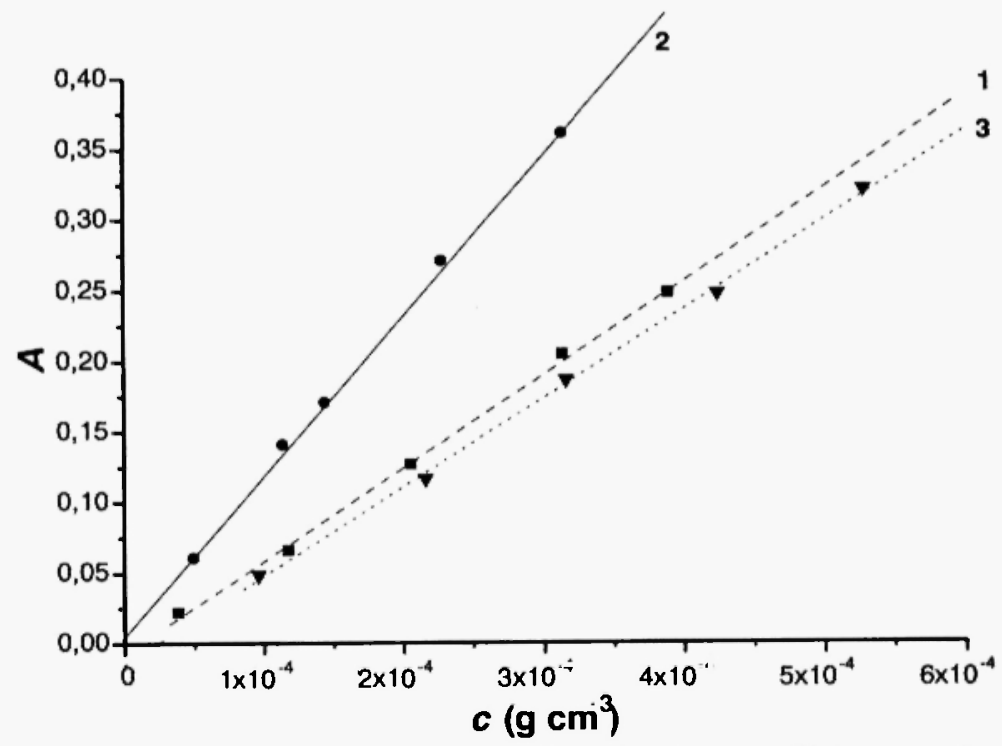

Fig. 5: Experimental determination of extinction coefficients; spherical silica particles, $a=2.5 \mu \mathrm{m}$. Dispersing medium: 1 (口) $\mathrm{NaN}_{3}$

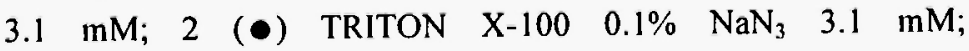
$3(\nabla) \operatorname{SDS} 3.110^{-5} \mathrm{M}$ 
critical micellar concentration. The indirect proof has been given by "fluorimetric" measurements performed at the same wavelength used for the turbidity measurements. The incident and read wavelength values were set at $330 \mathrm{~nm}$, where neither absorption nor fluorescence was, in fact, expected. In Figure 6 (adapted from $/ 2 /$ ) the signals collected in the fluorimeter for the

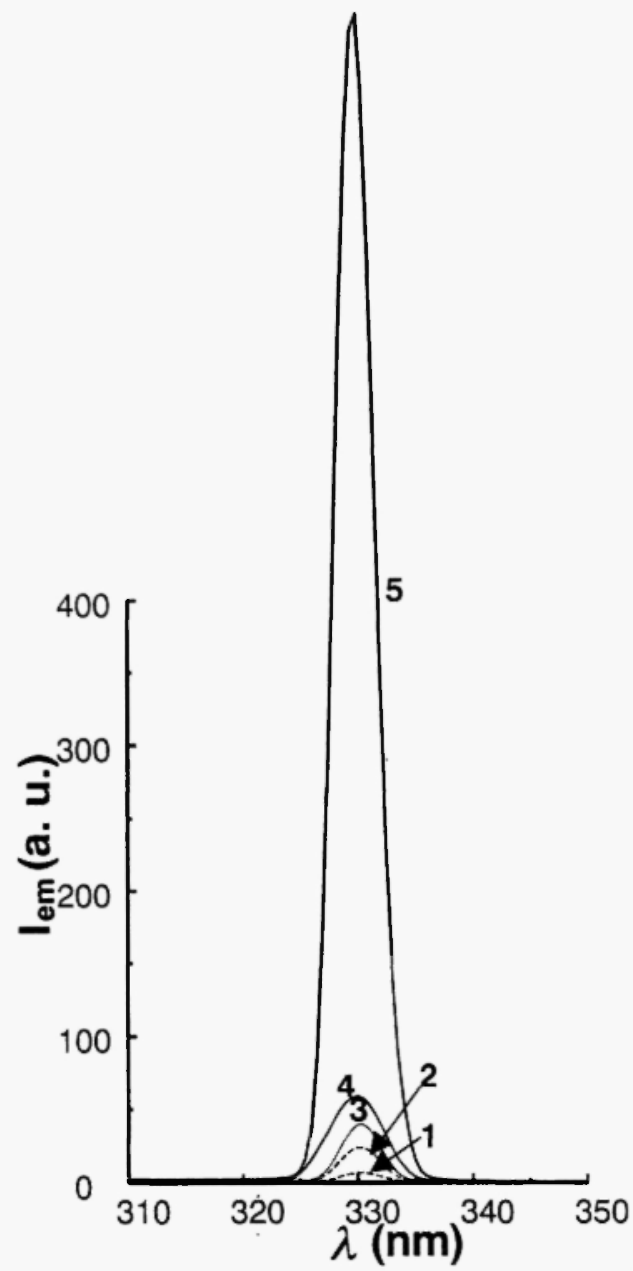

Fig. 6: Light emission (fluorimetric readings) at $90^{\circ}$ for dispersions of spherical, silica particles, $a=2.5 \mu \mathrm{m}$. Incident and reading wavelength=330 nm. 1: $\mathrm{NaN}_{3} 3.1 \mathrm{mM}$ (blank); 2: TRITON X-100 $0.1 \% \mathrm{NaN}_{3} 3.1 \mathrm{mM}$ (blank); 3: silica, $132 \mu \mathrm{g} \mathrm{cm}^{-3}$ in $\mathrm{NaN}_{3} 3.1 \mathrm{mM}$; 4: silica $124 \mu \mathrm{g} \mathrm{cm}^{-3}$ in $1.7 \mathrm{mM}$ sodium dodecyl sulphate (SDS) $/ \mathrm{NaN}_{3} 1.4 \mathrm{mM}$; 5: silica, $124 \mu \mathrm{g} \mathrm{cm}^{-3}$ in TRITON X-100 0.1\% and $\mathrm{NaN}_{3} 3.1 \mathrm{mM}$. (a.u.): arbitrary units. 
three silica samples are plotted. The signals for the pure solutions without dispersed samples are also reported (blanks, lines 1-2). It is evident that a relatively large amount of light is scattered at $90^{\circ}$ only when TRITON X-100 $0.1 \%$ is added. We conclude that the high adsorption of TRITON X-100 on silica particles may be responsible for the different optical behavior of the analyzed dispersions when this surfactant is added.

From these studies it can be seen that it is important to know the effects of the mobile phase (the dispersing medium) when flow-assisted separation techniques are applied to dispersed samples. In FFF, for instance, we have shown that the mobile phase composition plays an active role in modulating retention $/ 15,16 /$. In this review we want to emphasize that it can also modify the detector response. Therefore, if we want to apply the modeling approach for the evaluation of the extinction coefficient of dispersed samples when separated through flow-assisted techniques (for instance, for PSAD analysis in FFF) care must be taken in the choice of the mobile phase composition in order to maintain the extinction coefficients at their predicted values.

\section{Evaluation of the extinction coefficient by the modeling approach}

The modeling approach proposed for the prediction of the extinction efficiency $(Q)$ has been experimentally verified by us by comparing theoretically predicted and experimental values of $Q / 4 /$. In Figure 7 (adapted from /4/) are reported the predicted profiles for the extinction efficiency $(Q)$ function at null acceptance angle $\left(\theta=0^{\circ}\right)\left(Q_{0}\right)$ and at the acceptance angle of the employed UV-Vis detector, as a function of $a / \lambda$, calculated for the relative refractive index of polystyrene, compact particles $(\mu F 1.18)$. The acceptance angle of the detector has been accurately determined by measuring the diameter of the photodiode and its distance from the cell and found to be $\theta=4.6^{\circ}$. In the same figure, the experimental points of $Q$ relative to polystyrene particles of two different diameters are also plotted. Evaluation of the experimental $Q$ values has been performed by plotting $T$ values (at different $\lambda$ ) for a set of polystyrene standard particles samples of given diameter at different concentrations. As we have shown, from such measurements the experimental extinction coefficient $(\mathcal{K})$ values can be obtained from the slope of linear regression analyses $/ 1,2 /$. Experimental extinction efficiency values $(Q)$ can be then determined by applying Eq. 15 .

Good agreement between experimental and predicted $Q$ values can be observed in a range where they tend to be constant and close to unity. 


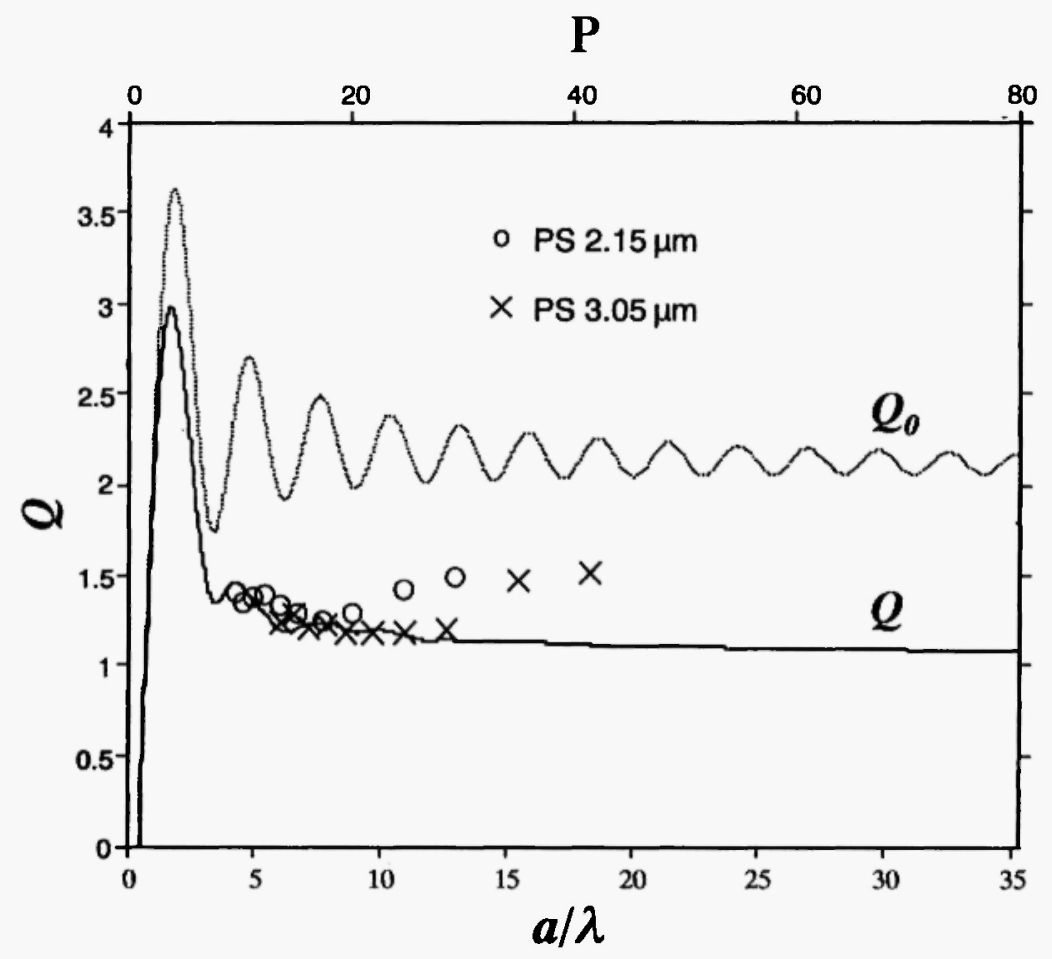

Fig. 7: Comparison between predicted (full line $Q$; dotted line $Q_{0}$ ) and experimental extinction efficiency values $(\mathrm{O}: \mathrm{PS}=2.15 \mu \mathrm{m}$; $\mathrm{X}: \quad \mathrm{PS}=3.05 \mu \mathrm{m})$. Acceptance angle $\theta=4.6^{\circ}$, $\lambda$ (in water) $=165-500 \mathrm{~nm}$

However, deviations from theory are evident with increasing $a / \lambda$, that is when light absorption of polystyrene particles begins to occur for a selected value (in air) of incident wavelength $\lambda<370 \mathrm{~nm}$. From this finding it is evident that a constant extinction efficiency approximately equal to 1 can be predicted only when particles do not absorb light. As previously discussed in PART I, it must be noted that constant, experimentally determined $\mathcal{K}$ values are actually average values with respect to the PSD of the dispersed sample. Therefore, it is important to bear in mind that experimental extinction efficiency values $(Q)$ determined by the proposed calibration approach must be also considered as average values. We have already pointed out, however, that the modeling approach holds true if half of the volume-surface average diameter $\left(D_{32}\right)$ is assumed for particle radius $(a) / 7 /$. As a consequence, the 
average $Q$ values obtained from average, experimental $K$ values can be considered valid for particle diameters corresponding to their $D_{32}$ mean values.

\section{Particle size and sample mass distribution in field-flow fractionation}

Among possible applications of the method described in this review, we have particularly dealt with the determination of PSAD of micronsize particles by FFF. We have applied the procedure to PSAD in either gravitational FFF (GFFF) and flow FFF (FIFFF) $2,4,17$. For both GFFF and FlFFF, conversion of the detector signal in the fractogram can be performed with $\mathcal{K}$ values obtained through either the calibration approach (Eq. 23) or the modeling approach (Eq. 20). We will show here examples of PSAD obtained using both approaches for the evaluation of $\mathcal{K}$ On the other hand, differences between GFFF and FIFFF lie in the required conversion of the fractogram from retention volume to particle size values.

The procedure to convert retention volume to particle size values in GFFF has been developed and tested $2,8,18,19$ /. This topic stands beyond the matter discussed in this review. An example of GFFF/PSAD with the experimental determination of $\mathcal{K}$ by the calibration approach is reported in Figure 8 (adapted from $/ 2 /$ ). Conversion of the fractogram was performed by means of Eq. 23. We observe that, even if the experimental value for the extinction coefficient entered to determine the PSAD curves is an average value, the dependence of this parameter on particle diameter is taken into account in the derivation, through Eq. 20 , of the final expression for $f_{\text {in.l }}(d)$ in Eq. 23. This dependence is actually expressed in the product between the turbidity value corresponding to each $i$ th digitized point of the fractogram $\left(T_{1}\right)$ and the relative diameter value $d_{i}$. Therefore, experimental average values of the extinction coefficient determined by the calibration approach can be effectively used to obtain the PSAD function. An example of GFFF/PSAD with the prediction of $\mathcal{K}$ values by the modeling approach is reported in Fig. 9 (adapted from $/ 7 /$ ). Here conversion of the fractogram is performed through Eq. 20.

The same conversion through Eq. 20, that is with the prediction of $\mathcal{K}$ values by the modeling approach, is used in Fig. 10 (adapted from /17/) where an example of FIFFF/PSAD is reported for the same silica sample the GFFF/PSAD of which is reported in Fig. 8. The main advantage of FIFFF 


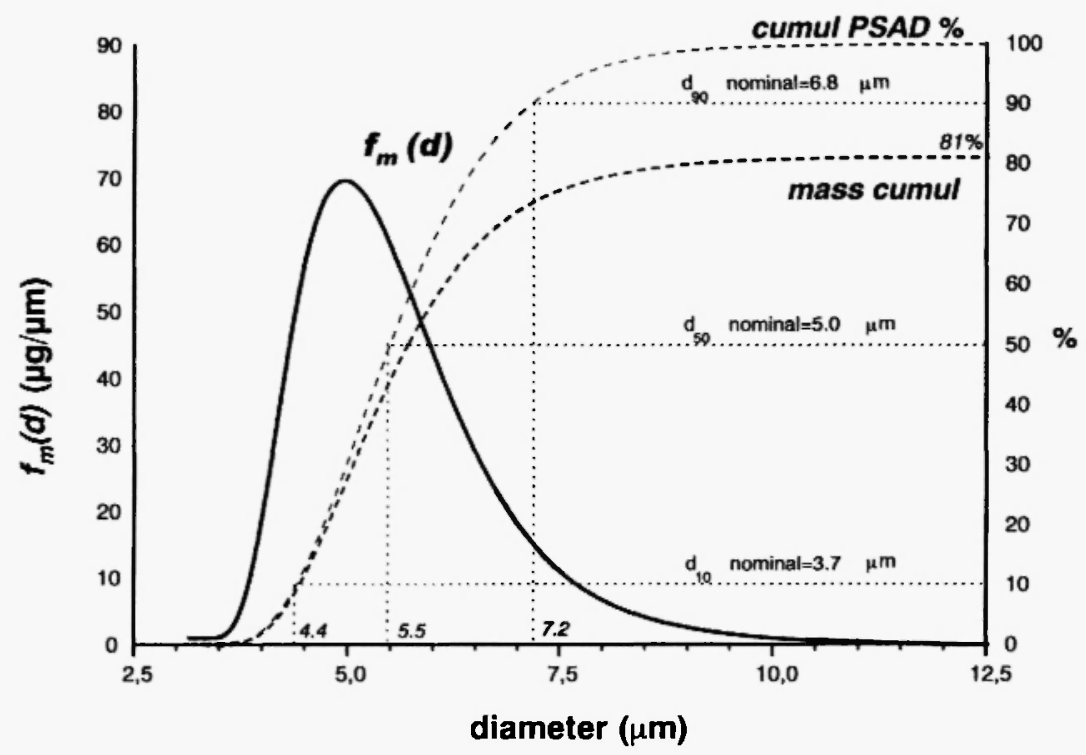

Fig. 8: GFFF/PSAD $\left(f_{m}(d)\right.$, full line); cumulative, size-amount percent distribution (cumul PSAD\%, dashed line); cumulative mass distribution (mass cumul, dashed line) and size distribution percentiles $\left(d_{10}, d_{50}, d_{90}\right)$; sample: spherical silica particles (LiChrospher $5 \mu \mathrm{m}$, Merck). Injected amount: $200 \mu \mathrm{g}$; mobile phase: TRITON X-100 $0.1 \% / \mathrm{NaN}_{3} 3.1 \quad 10^{-3} \mathrm{M}$; experimental $\mathcal{K}$ value $=$ $1180 \mathrm{~cm}^{2} \mathrm{~g}^{-1}, \lambda=330 \mathrm{~nm}$

with respect to GFFF lies in the fact that retention in FIFFF is reported to depend on size only /13/. In FIFFF the conversion of retention volume to size values of micronsize particles is, thus, relatively straightforward once the retention volume axis has been calibrated through the FIFFF elution of standard, micronsized spherical particles of known size. In Figure 10 a) the fractogram obtained for the silica sample is superimposed on the fractogram of a mixture of five polystyrene standards, eluted at the same channel flow rate, field strength and mobile phase composition. FIFFF of particle standards is a required procedure for the calibration in size of the retention axis, also described by us in $/ 17 /$. From Figure 10 a) a second advantage of FIFFF with respect to GFFF is evident: the very short analysis time, which is about onethird of the analysis time required for the GFFF/PSAD of the same sample, reported in Fig. 8. 


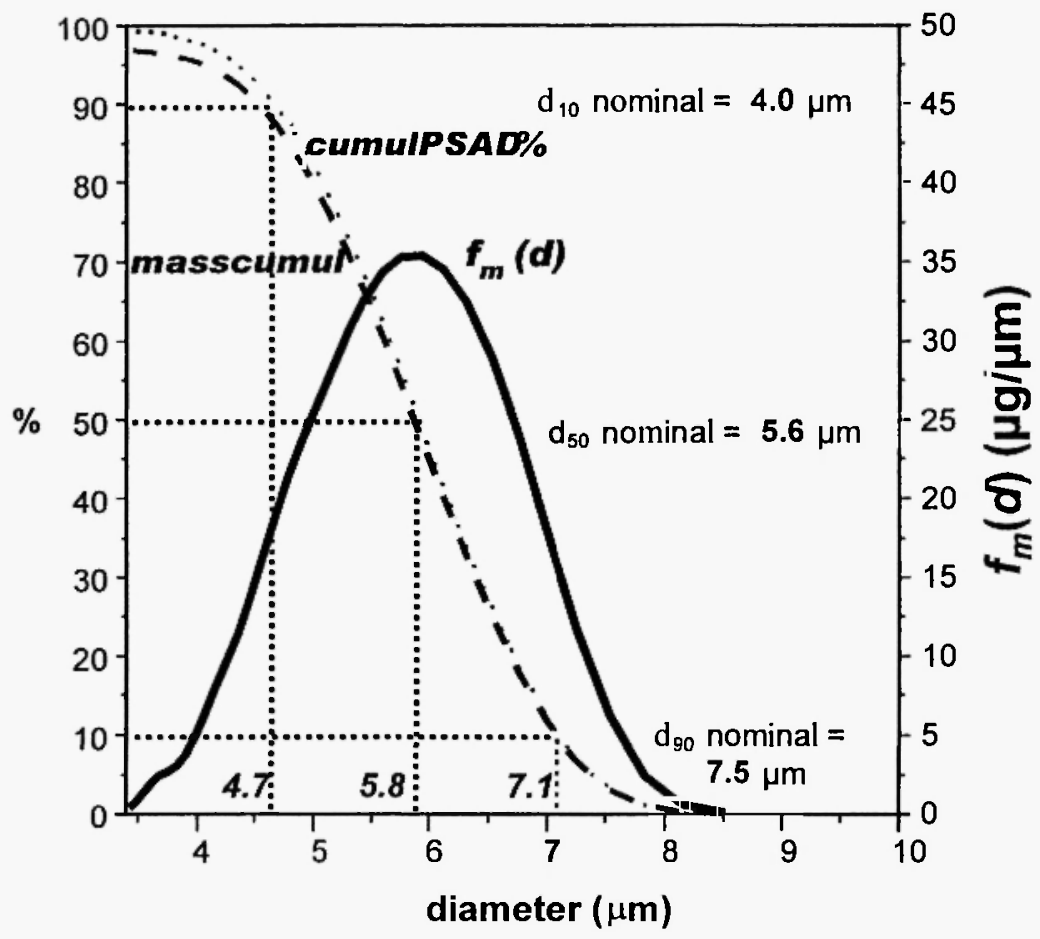

Fig. 9: GFFF/PSAD; sample: derivatized, spherical silica particles (LiChrospher RPI8e $5 \mu \mathrm{m}$, Merck); mobile phase: SDS $1 \% \mathrm{w} / \mathrm{v}$; $\lambda=330 \mathrm{n} / \mathrm{n}$

In all figures relevant to PSAD analysis by FFF (Figs. 8-10), the dashed lines are the cumulative size distributions (cumuIPSAD\%) and cumulative mass distribution curves (masscumul), which are simply obtained from integration of $f_{m}(d)$. The cumulative size distribution profile gives immediately the experimental distribution percentiles, which can be compared with the nominal values of the samples. The nominal values of $d_{10}$, $d_{50}$ and $d_{90}$ were obtained by the manufacturer (Merck, Darmstad, D) by means of a CILAS 850 (CILAS, Orleans, F) Laser Diffraction Particle Size Analyzer. The accuracy of the experimental percentiles is generally acceptable. In fact, it must be pointed out that differences in percentile values as high as $10 \%$ are commonly reported when different techniques for PSD studies are compared. From the cumulative mass distribution profile it is also easy to evaluate the total sample recovery. This is done by dividing the 

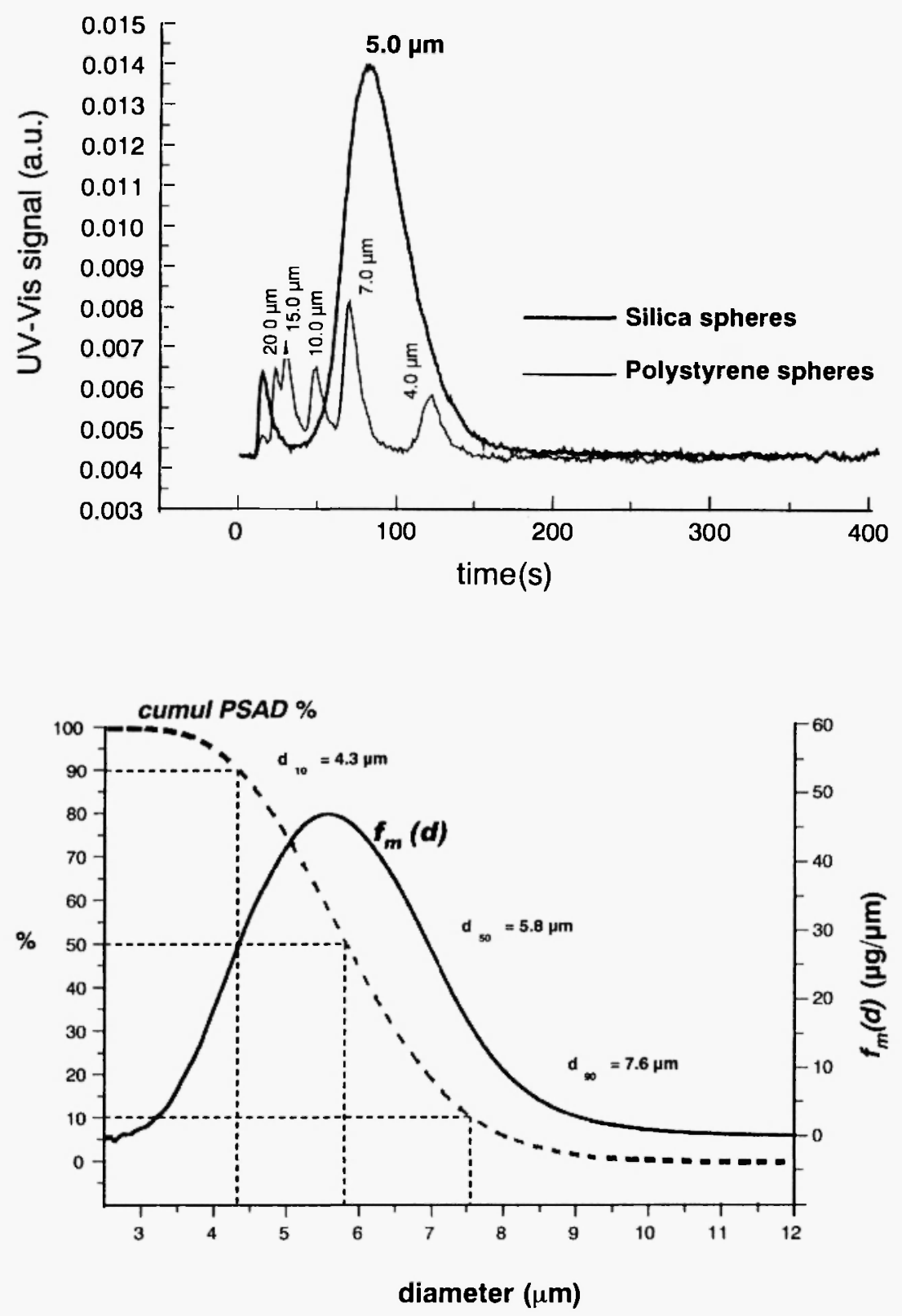

Fig. 10: FIFFF/PSAD; sample: spherical silica particles (LiChrospher $5 \mu \mathrm{m}$, Merck); mobile phase: $0.01 \%$ TRITON X-100, 0.02\% $\mathrm{NaN}_{3}$; cross-flow rate $=2.3 \mathrm{ml} / \mathrm{min}$, channel flowrate $=6.0$ $\mathrm{ml} / \mathrm{min}$. a) Retention comparison with five polystyrene standard particles; b) PSAD; $\lambda=330 \mathrm{~nm}$ 
asymptotic limit of this curve by the known injected quantity. The accurate, single-run evaluation of sample recovery in FFF is, hence, immediately obtained. No other procedure can give sample recovery in FFF in the same way $/ 13 /$.

\section{CONCLUSIONS}

The approach described here shows that quantitative analysis of dispersed samples separated by flow-assisted techniques is possible with standard UV$V$ is detectors. It is based on a simple relationship between signal and concentration, the proportionality constant of which can be predicted within known limiting conditions. Within these limits, quantitative analysis can be obtained from single runs with no need for calibration, except una tantum instrumental calibrations. However, the approach has been limited up to now to micronsized particulate samples. We are currently developing some applications for real samples of different origin, from starch granules to cells and bacteria. Within the field of bio-applications, the interest in rapid and cost-effective methods for bacteria quantification is increasing. Enumeration of bacteria is, for instance, an important quality control of bio-process developments in whole-bacteria vaccine production. Information about the total amount of bacteria is also of prime importance from medicine to wastewater plant process monitoring. A range of techniques is available for the direct and indirect detection and determination of bacteria. By coupling FFF to UV-Vis turbidity we are obtaining promising results, as in the case of the limit of detection of fractionated (and, thus, purified) bacteria. We have been finding limits of detection values in the range of the most recently proposed methods based on polymerase chain reaction, and lower than in flow cytometry.

We hope that our method for quantitative analysis of dispersed sample in flow-assisted separation techniques could soon become of broader use than simply for FFF, since other flow-assisted separation techniques are now entering this field.

\section{PERSPECTIVES}

The modeling approach to determine the extinction efficiency of dispersed samples carried through a standard UV-Vis detector has up to now 
employed a restricted model to describe the extinction efficiency of particles. Such a model holds true only for micronsize particles. Therefore, only for such samples the direct conversion from signal to sample mass or concentration is possible by single-run analysis without previous calibration using standards. Other models are available to predict the extinction properties of dispersed particles of any size, like the Mie theory for scattering. However, they all require the exact knowledge of many sample characteristics, like size, shape, refractive index, density, and of detector optics (i.e. the acceptance angle). These requirements are a severe drawback because they are unlikely to be available for complex samples.

We are currently working on a new method of quantitative analysis of dispersed samples for those flow-assisted separation techniques that are able to give, alone or coupled with specific uncorrelated techniques, the size distribution of the sample. By this method we hope to obtain the optical properties of the analyte without calibration using standards and without the use of a model requiring knowledge of sample characteristics and detector optics. This should make the quantitative approach to flow-assisted separation of dispersed samples from single-run analysis quite straightforward. In fact, this method seems to give the PSAD independently if just the relationship between retention volume and particle size is either theoretically or empirically available, as in the case of FFF. For this new method the use of a diode-array UV-Vis detector (UV-Vis/DAD) is a necessary instrumental requisite to calculate the sample extinction efficiency in real-time, from a single-run analysis. The algorithm we are developing is based on the fundamental property of the extinction efficiency of particles, which is a function of the ratio between particle size and incident wavelength 13/. The solution independently gives the extinction efficiency function and the PSAD by non-linear least-squares fitting. We are currently testing this new method by simulation of different size distribution and extinction efficiency functions. The algorithm seems to be accurate and rugged. We hope to apply it soon to real signals. If our expectations are fulfilled, this new approach could represent a general solution to quantitative analysis of dispersed samples by flow-assisted techniques using UV-Vis turbidity detection. 


\section{ACKNOWLEDGEMENTS}

The authors wish to thank P.S. Williams, The Cleveland Clinic Foundation, for manuscript revision. Work supported by the European Commission, 4th Framework Program, INCO-Copernicus, Contract No. ERB ICI5-CT98-0909.

\section{REFERENCES}

I P. Reschiglian, D. Melucci and G. Torsi, Chromatographia, 44, 172 (1997).

2 P. Reschiglian, D. Melucci, A. Zattoni and G. Torsi, J. Microcolumn Sep., 9, 545 (1997).

3 van de Hulst, in: Light Scattering by Small Particles, ed. by H.C. van de Hulst, Dover Publications Inc., New York, 1981.

4 A. Zattoni, D. Melucci, G. Torsi, P. Reschiglian, J. Chromatogr. Sci., 38, 122 (2000).

5 G. Torsi, G. Chiavari, C. Laghi, A. M. Asmundsdottir, F. Fagioli, and R. Vecchietti, J. Chromatogr., 482, 207 (1989).

6 G. Torsi, G. Chiavari, C. Laghi and A. M. Asmundsdottir, J. Chromatogr., 518, 135 (1990).

7 T. Kurti, J.F. MacGregor, in: Particle Size Distribution II, Assessment and Characterization, ACS Symposium 472, ed. by T. Provder, American Chemical Society, Washington D.C., 1991, ch.3.

8 P. Reschiglian, D. Melucci, G. Torsi, A. Zattoni, Chromatographia, 51, 87 (2000).

9 F.P. Hochgeshong, in: Treatise on Analytical Chemistry, ed. by I.M. Kolthoff and P.J. Elwing, Wiley Interscience, New York, 1964, Vol.5, Part I, p. 3289.

10 P. Walstra, Brit. J. Appl. Phys., 15, 1545 (1964).

11 P. Walstra, Brit. J. Appl. Phys., 16, 1187 (1965).

12 J.R. Hodkinson and I. Greenleaves, J. Opt. Soc. Amer., 53, 577 (1963).

13 Field-Flow Fractionation Handbook, ed. by M.E. Schimpf, K. Caldwell and J.C. Giddings, Wiley Interscience, New York, 2000.

14 P. Reschiglian and G. Torsi, Chromatographia, 40, 467 (1995). 
15 P. Reschiglian, A. Zattoni, D. Melucci, C. Locatelli and G. Torsi, in: Recent Res. Devel. Applied Spectroscopy, ed. by S.G. Pandalai, Research Signpost, Trivandrum, India, 2000, vol.3, p. 61.

16 P. Reschiglian, D. Melucci and G. Torsi, J. Chromatogr. A, 740, 245 (1996).

17 P. Reschiglian, D. Melucci, A. Zattoni, L. Mallo, M. Hansen, A. Kummerow and M. Miller, Anal. Chem., 72, 5945 (2000).

18 D. Melucci, G. Gianni, G. Torsi, A. Zattoni and P. Reschiglian, J. Liq. Chromatogr. R. T., 20, 2615 (1997).

19 P. Reschiglian, in: Encyclopedia of Chromatography, ed. by J. Cazes, Marcel Dekker, New York, 2001, ch. 195, p. 585.

\section{LIST OF SYMBOLS}

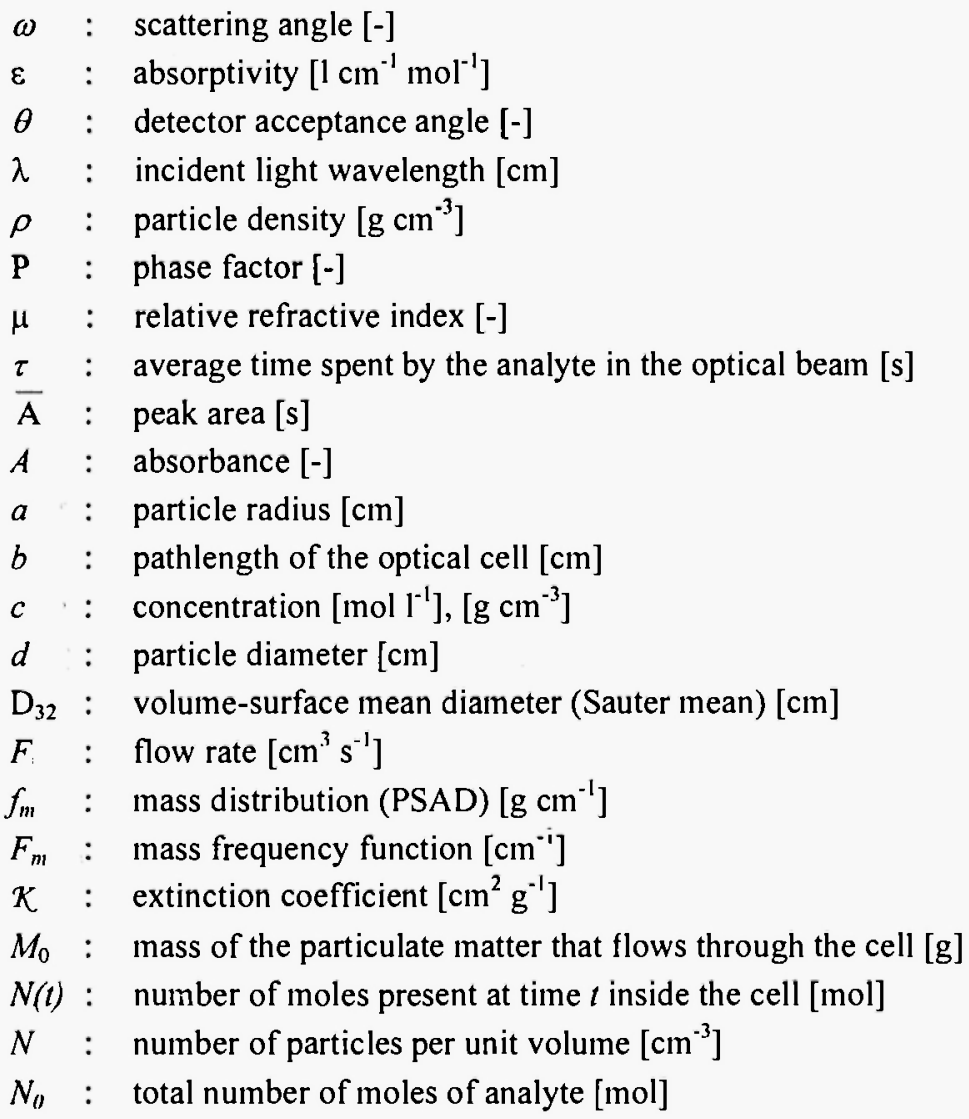


$N_{0} \quad$ : total number of moles of analyte [mol]

$P, P_{0}: \quad$ light radiant power $\left[\mathrm{erg} \mathrm{s}^{-1} \mathrm{~cm}^{-2}\right]$

$Q \quad: \quad$ extinction efficiency [-]

$Q_{0}:$ extinction efficiency for $\theta=0[-]$

$S:$ cross section of the detector cell $\left[\mathrm{cm}^{2}\right]$

$t$ : time [s]

$T:$ turbidity $\left[\mathrm{cm}^{-1}\right]$

$V_{r}:$ retention volume $\left[\mathrm{cm}^{3}\right]$ 
